\title{
Excited-State Dynamics in the Enhanced Green Fluorescent Protein Mutant Probed by Picosecond Time-Resolved Single Photon Counting Spectroscopy
}

\author{
Mircea Cotlet, ${ }^{\dagger}$ Johan Hofkens, ${ }^{*}, \dagger$ Michael Maus, ${ }^{\dagger}$ Thomas Gensch, ${ }^{\dagger}$ Mark Van der Auweraer, ${ }^{\dagger}$ \\ Jan Michiels, $;$ Gunter Dirix, ${ }^{*}$ Maarten Van Guyse, $*$ Jos Vanderleyden, \\ Antonie J. W. G. Visser, ${ }^{\dagger} \S$ and Frans C. De Schryver ${ }^{\dagger}$ \\ Department of Chemistry, Katholieke Universiteit Leuven, Celestijnenlaan 200 F, 3001 Heverlee, Belgium, \\ Department of Agriculture, Katholieke Universiteit Leuven, Kardinaal Mercierlaan, 3001 Heverlee, Belgium, \\ and Department of Biomolecular Sciences, Wageningen Agricultural University, Dreijenlaan 3, \\ 6703 HA Wageningen, The Netherlands
}

Received: October 19, 2000; In Final Form: March 8, 2001

\begin{abstract}
The complex photophysics of the wild-type green fluorescent protein (GFP), one of the most popular fluorescent probes in biology, has been extensively documented in literature. The excited-state dynamics of GFP was explained by means of a model implying excited-state proton transfer (ESPT) and three forms of the chromophore, a protonated A form absorbing at $400 \mathrm{~nm}$ and two deprotonated I and B forms absorbing at around $475 \mathrm{~nm}$. We report here a systematic picosecond time-resolved fluorescence study of the enhanced green fluorescent protein (EGFP) variant, carrying the Ser65-Thr and Phe64-Leu mutations. By means of multiple excitation wavelength time-resolved experiments, we were able to distinguish between the fluorescence decay times of the deprotonated $\mathrm{I}^{*}$ and $\mathrm{B}^{*}$ states (3.4 and $2.7 \mathrm{~ns}$ ). Spectrally, we found the I form being red shifted in comparison with the B form, both in absorption and in emission. Evidence for an excited-state reaction, namely, proton transfer, is also reported. An additional protonated species is proposed in the photophysical scheme in order to explain the excited-state dynamics of EGFP on the basis of our results as well as previous reported data. Two alternative models are presented, both of them applicable also to the data reported in relation with wild-type GFP.
\end{abstract}

\section{Introduction}

In recent years, the green fluorescent protein (GFP) together with its mutants have become one of the most used fluorescent probes in cell biology and molecular biology. ${ }^{1-3}$ Nowadays GFP is used in a large number of applications as a noninvasive fluorescent marker for gene expression and protein localization in cells and organisms or in protein-protein interaction studies via fluorescence resonant energy transfer. ${ }^{4-6}$

The usefulness of GFP as a biological marker is due to the fact that the chromophore responsible for absorption and fluorescence in the visible region is formed via an intramolecular biosynthesis from the tripeptide Ser65-Tyr66-Gly67 in the absence of any external cofactor, ${ }^{7,8}$ allowing the fusion of the DNA sequence of GFP with that of any other nonfluorescent protein that can be later investigated with a common fluorescence technique. Moreover, because the chromophore itself is rigidly encapsulated inside an 11-stranded $\beta$-barrel amino acid structure, ${ }^{9-11}$ eventual nonradiative cis - trans isomerization is prevented and fluorescence is emitted with a high quantum yield. Genetic manipulation of the amino acid residues implied in the formation of the chromophore can lead to mutants with improved photostability and modified spectral properties, $, 8,12,13$ one interesting variant, especially for biological applications using fluorescence microscopy, being the enhanced green

* To whom correspondence should be addressed. Fax: +32(016) 327990 E-mail: johan.hofkens@chem.kuleuven.ac.be.

† Department of Chemistry, Katholieke Universiteit Leuven.

$\doteqdot$ Department of Agriculture, Katholieke Universiteit Leuven.

$\S$ Wageningen Agricultural University. fluorescent protein (EGFP) which carries the Ser65 $\rightarrow$ Thr and Phe64 $\rightarrow$ Leu mutations

The increased interest in these proteins requires systematic and careful study of the photophysical and photochemical properties and several publications on the basis of stationary and time-resolved spectroscopic experiments in relation to the high-resolution three-dimensional structure already reported an important part of their complex photophysical behavior. ${ }^{14-26}$ Tsien and co-workers ${ }^{12}$ associated for the first time the two absorption peaks from 398 and $475 \mathrm{~nm}$ of wild-type GFP, whose ratio is sensitive to factors as temperature, $\mathrm{pH}$, ionic strength, and illumination with blue light, $8,14,24,25$ with the presence of the chromophore in two stable and interconvertible forms: a protonated A and a deprotonated B form. Later on, in a landmark publication based on femtosecond fluorescence upconversion experiments, Boxer and co-workers ${ }^{14}$ proposed a general photophysical scheme based on three forms of the chromophore and implying excited-state proton transfer (ESPT). In their opinion, the strong green fluorescence of the wild-type GFP, when excited with blue light, is the final result of a fast ESPT taking place in about $4 \mathrm{ps}$ from the excited $\mathrm{A}^{*}$ form to an intermediate deprotonated excited form $\mathrm{I}^{*}$, supposed to be an unrelaxed form of $\mathrm{B}^{*}$. The $\mathrm{I}^{*}$ state will relax mainly radiatively to the corresponding ground-state I or, rarely, to the $\mathrm{B}^{*}$ form. To a similar conclusion arrived also Lossau et al. ${ }^{15}$ These three forms were identified later in the ground state, together with their $0-0$ transitions, by spectral hole burning experiments at cryogenic temperature ${ }^{18}$ as well as in the excited state by picosecond time-resolved fluorescence experiments, and fluo- 
rescence decay times of 3.3 and 2.8 ns for the I and B forms, respectively, were attributed. ${ }^{16}$

On the basis of the three-dimensional structure resolved with atomic resolution, the existence of the above-mentioned forms of GFPs chromophore as well as the mechanism of the ESPT has been extensively discussed at the molecular level by Brejc et al. ${ }^{11}$ by taking into account the existence of two different hydrogen bond networks between the chromophore and the surrounding amino acid residues in wild-type GFP. The proposed model explained also the reversible photoconversion of wild-type GFP under irradiation with $400 \mathrm{~nm}$ light.

The present study focuses on the excited-state dynamics of the EGFP mutant. In this variant, the replacement of serine by threonine in position 65 leads to a major increase and red shift to $488 \mathrm{~nm}$ of the absorption peak associated with the deprotonated form of the chromophore. The protonated state is, compared to the natural protein, less populated, and the mutant displays, upon excitation at $488 \mathrm{~nm}$, an almost identical but more intense emission spectrum and higher photostability. ${ }^{8,13}$ Despite the large interest manifested in biological applications for this mutant, there have been relatively few studies referring to its photophysical properties. ${ }^{15,17,19,23,27,28}$ Lossau et al. ${ }^{15}$ reported the detection of a 10 ps component associated with ESPT in EGFP and adopted a similar three-level scheme as for wild-type GFP. The presence of a proton transfer was also reported by Webb and co-workers ${ }^{23}$ as well as by Rigler and co-workers ${ }^{27,28}$ using fluorescence correlation spectroscopy in $\mathrm{pH}$ dependence studies. The three forms A, I, and B and their 0-0 transitions were identified for a closely related mutant (only carrying the Ser65-Tyr point mutation) with hole burning spectroscopy. ${ }^{19}$

\section{Materials and Methods}

Purification of EGFP. Standard methods were used for in vitro DNA manipulations. ${ }^{29}$ The gene coding for EGFP was first removed from pEGFP (Clontech) as an 800 bp EcoRIPstI fragment and subsequently inserted into $\mathrm{pBAD} / \mathrm{His} \mathrm{A}$ (Invitrogen). The expression of the protein was induced for 12 h in Escherichia coli Top10 cells grown at an optical density at $600 \mathrm{~nm}$ of 0.5 using $0.2 \%$ arabinose. The polyhistidine protein was subsequently isolated and purified under native conditions by Ni-chelation chromatography using the Xpress Protein Purification System as recommended by the manufacturer. Purity of the samples was confirmed by polyacrylamide gel electrophoresis. Finally, the proteins were concentrated in a Vivaspin 6 concentrator.

Spectroscopy. All spectroscopic measurements were performed at room temperature (293 K). EGFP was dissolved in a $10 \mathrm{mM}$ sodium phosphate buffer $(\mathrm{pH} 7.4$, concentration of about $10^{-7} \mathrm{M}$ ) and stored into a $10 \mathrm{~mm}$ path length quartz cuvette.

Steady-state absorption spectra were measured using a PerkinElmer Lambda 40 double-beam spectrophotometer with $2.0 \mathrm{~nm}$ resolution. Steady-state fluorescence excitation and emission spectra were measured using a Spex Fluorolog 1500 spectrophotometer (1.0 $\mathrm{nm}$ resolution). Fluorescence was collected in a right angle geometry. The recorded fluorescence excitation and emission spectra were corrected according to literature procedures. ${ }^{30}$ Time-resolved fluorescence measurements were performed using an experimental setup described elsewhere. ${ }^{31}$ Briefly, laser excitation was achieved by frequency doubling (from 400 to $543 \mathrm{~nm}$ ) or tripling $(270 \mathrm{~nm}$ ) the output of an actively mode-locked picosecond Ti:sapphire laser (Tsunami Spectra Physics; $82 \mathrm{MHz}$ repetition rate) pumped by an $\mathrm{Ar}^{+}$ laser (BeamLok 2080 Spectra Physics) and then reducing the repetition rate to $8.13 \mathrm{MHz}$ into an acousto-optical modulator (Pulse Selector 3980 Spectra Physics). Laser pulses were passed through a Berek polarization compensator (New Focus 5540) and a Glan polarizer in order to obtain vertically polarized excitation light at the sample. Fluorescence was collected in a right angle geometry. Spectral separation was performed with a stepper motor controlled double monochromator (Sciencetech 9030) with $5 \mathrm{~nm}$ resolution and detected with a cooled MCPPMT (Hamamatsu E 3059-500). An additional polarizer was inserted in the emission path, oriented at magic angle $\left(54.7^{\circ}\right)$ with respect to the excitation polarization. The signal from the MCP-PM was amplified in a fast rise time dual amplifier (Hewlett-Packard 8447 D) and input into a time-correlated single photon counting PC card (SPC 430, Picoquant GmbH). Triggering was possible by sending $20 \%$ of the excitation laser beam to a nanosecond photodetector (Newport 818-BB-21). The experimental instrumental response function (IRF) for all excitation wavelengths was in the $40-80$ ps range. For each excitation wavelength, several decay curves at different emission wavelengths were collected in 4096 channels and with about 10000 detected counts at the maximum of the fluorescence signal. Time-resolved spectra using excitation at $400 \mathrm{~nm}$, with a $10 \mathrm{~nm}$ step, were recorded from 400 up to $600 \mathrm{~nm}$ by timegating the single photon counting detection system and scanning the emission spectrum. Correction for the MCP-PMT spectral sensitivity was taken into account. Before and after each experiment, the sample was checked regarding photostability by recording the absorption spectrum.

Data Analysis. The time-resolved fluorescence decays recorded for each excitation wavelength were globally analyzed over the emission spectrum and different time windows with a homemade program that uses the $\delta$ convolution method. ${ }^{32-34}$ This method allows correction for wavelength dependence of the shape of the IRF and requires the measurement of the timeresolved fluorescence of a reference compound in the same experimental conditions as those used for the investigated sample (i.e., the same excitation, emission wavelength, and laser power). The reference compound must exhibit a monoexponential behavior for the fluorescence decay curve described by a model function of the following type:

$$
F^{\mathrm{ref}}(t)=\alpha^{\mathrm{ref}} \exp \left(-t / \tau^{\mathrm{ref}}\right)
$$

where $F^{\text {ref }}$ is the fluorescence $\delta$ function, $\alpha^{\text {ref }}$ is a preexponential factor, and $\tau^{\text {ref }}$ is the fluorescence lifetime of the reference sample. The parameters of the measured fluorescence decay $\left(D^{\mathrm{s}}\right)$ of the sample can be obtained by performing an iterative convolution of the measured decay of the reference compound $\left(D^{\text {ref }}\right)$ with a modified model function $\left(F^{\mathrm{s}}\right)$ :

$$
D^{\mathrm{s}}=D^{\mathrm{ref}} \otimes F^{\mathrm{s}}
$$

where the modified model function is given by

$$
F^{\mathrm{s}}(t)=\sum_{j} \frac{\alpha_{j}^{\mathrm{s}}}{\alpha^{\mathrm{ref}}}\left[\delta(0)+\left(\frac{1}{\tau^{\mathrm{ref}}}-\frac{1}{\tau_{j}^{\mathrm{s}}}\right) \mathrm{e}^{-\left(t / \tau_{j}^{\mathrm{s}}\right)}\right]
$$

Here, $a_{j}=\alpha_{j}^{\mathrm{s}} / \alpha^{\text {ref }}$ and $\tau_{j}^{\mathrm{s}}$ are the preexponential factor and the fluorescent decay time of component $j$, and $\delta(0)$ is the Dirac delta function at time zero. Analysis of the decay curves with the above-presented method was possible using a home-written program which uses a reweighted iterative reconvolution method based on the Marquard algorithm. The contribution of the decay 

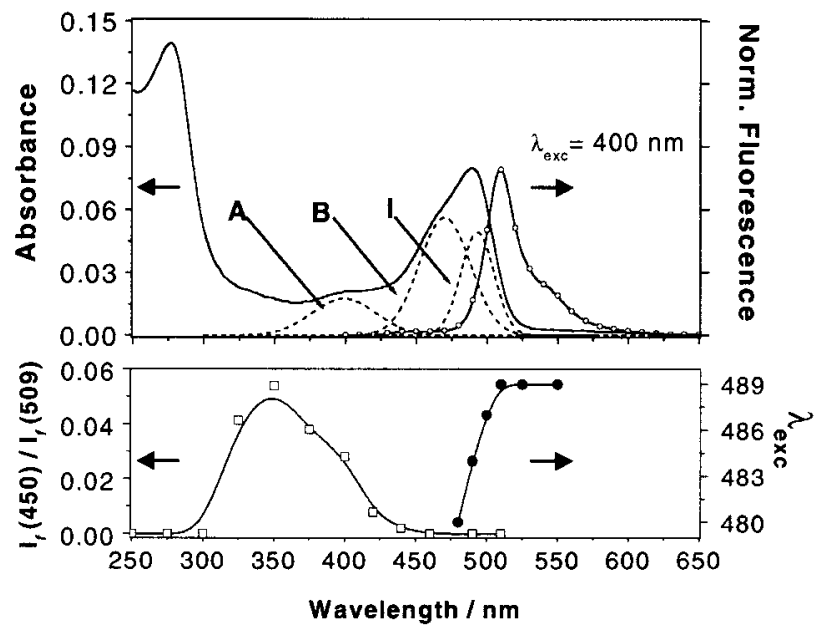

Figure 1. Upper panel: Steady-state absorption spectrum (solid line) with the corresponding decomposed bands A, B, and I (dashed line), using Gaussian functions; fluorescence spectrum (line + open circle) excited at $400 \mathrm{~nm}$. Lower panel, left part: The ratio of the fluorescence bands at 450 and $509 \mathrm{~nm}$ as a function of excitation wavelength (line + open square). Lower panel, right part: The maximum of the excitation spectrum $\left(\lambda_{\text {exc }}\right)$ as function of the detection wavelength (line + dots). The decomposition of the absorption spectrum in Gaussian bands was performed in order to get an estimate of the spectral position of the forms existing in EGFP for a better understanding of the forthcoming proposed models.

times recovered after the global analysis was estimated using the relative amplitudes:

$$
a_{j}^{\mathrm{rel}}=\frac{a_{j} \tau_{j}}{\sum_{j} a_{j} \tau_{j}}
$$

The goodness of the fits were judged by the values of the reduced $\chi^{2}$ as well as by inspecting the residual function graphs for each fitted data set. ${ }^{33}$ By globally analyzing fluorescence decay curves detected in different experimental conditions in a large number of channels and with high number of total counts, decay times differing with $10 \%$ or more can be recovered. ${ }^{33}$ As reference compounds, depending on the excitation wavelength, bis-[1-octadecylbenzthiazole-2-]-monomethyne perchlorate in methanol $\left(0.015 \mathrm{~ns}\right.$ lifetime $\left.{ }^{35}\right)$, bis-[1-octadecylbenzthiazole-2-]-trimethyne perchlorate in methanol ( 0.155 ns lifetime $\left.{ }^{35}\right)$, and erythrosine in water $\left(0.09\right.$ ns lifetime $\left.{ }^{35}\right)$ were used.

\section{Results}

Steady-State Spectroscopy. As previously reported, $8,13,23$ the absorption spectrum of EGFP (Figure 1) displays three major peaks in the UV-visible region: the 400 and $488 \mathrm{~nm}$ peaks, corresponding to the protonated and deprotonated forms of the chromophore, and the $275 \mathrm{~nm}$ peak, related to the absorption of the aromatic region of the protein. Decomposition of the visible region of the spectrum using Gaussian functions results in three bands with peaks located at about 400, 470, and 490 $\mathrm{nm}$ (bands A, I, and B in Figure 1).

The emission spectrum of EGFP is characterized, as previously reported, ${ }^{8,13}$ by a strong peak at $509 \mathrm{~nm}$, a pronounced shoulder at about $540 \mathrm{~nm}$ when excited in the absorption band of the deprotonated form (above $450 \mathrm{~nm}$ ), and a supplementary weak peak at $450 \mathrm{~nm}$ when excited in the absorption band of the protonated form (below $450 \mathrm{~nm}$ ). Green fluorescence can be detected even when excited in the aromatic region. The
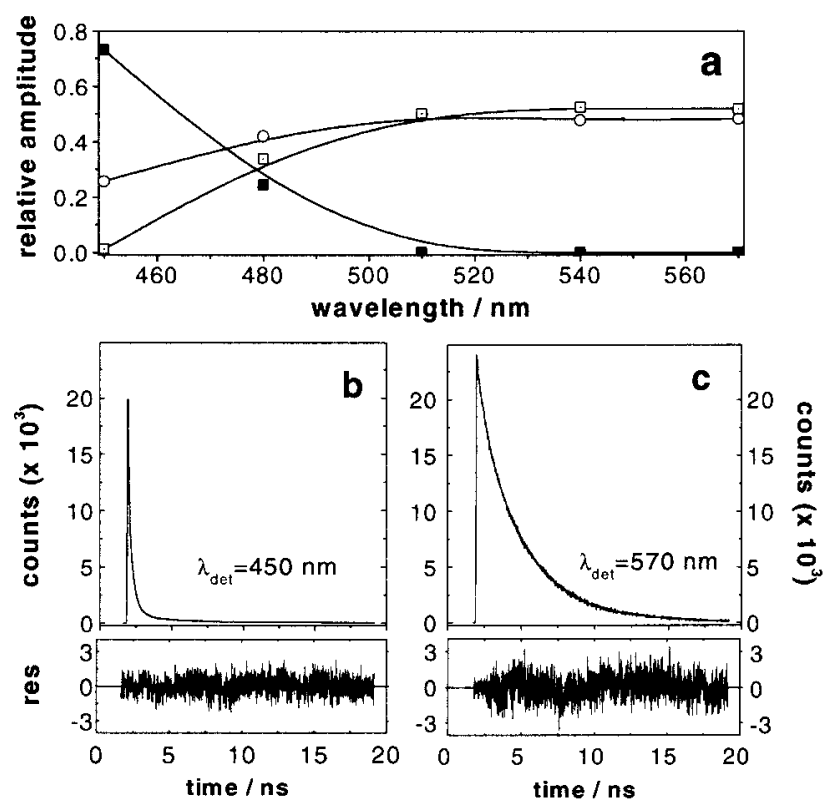

Figure 2. Global analysis results of the fluorescence decays of EGFP excited at $400 \mathrm{~nm}$ and detected from 450 to $570 \mathrm{~nm}$. (a) The relative amplitudes of the 0.25 (line + full square), 2.7 (line + open circle), and $3.4 \mathrm{~ns}$ (line + open square) decay times as a function of emission wavelength. (b) Fit (solid line) and residuals (lower panel) of the fluorescence decay detected at $450 \mathrm{~nm}$. (c) Fit (solid line) and residuals (lower panel) of the fluorescence decay detected at $570 \mathrm{~nm}$.

dependence of the relative intensity of the two fluorescence peaks (at 450 and $509 \mathrm{~nm}$ ) on the excitation wavelength is presented in the lower panel of Figure 1 and is the result of a decomposition analysis of the emission spectra using Gaussian functions.

The excitation spectrum of EGFP has a similar shape as the absorption spectrum and can also be decomposed in three Gaussian bands with peaks located at about 400, 470, and 490 $\mathrm{nm}$. When the excitation spectra at different detection wavelengths in the $480-550 \mathrm{~nm}$ range is monitored, the wavelength corresponding to the major peak in the excitation spectrum shifts from 480 to $489 \mathrm{~nm}$ by increasing the detection wavelength (Figure 1, lower panel). This shift can be regarded as a change in the relative contribution of the two Gaussian bands at 470 and $490 \mathrm{~nm}$, indicating the presence of at least two absorbing and emitting species in the spectral region of the deprotonated absorption band.

To check the occurrence of a possible photoconversion, we irradiated a sample of EGFP with $400 \mathrm{~nm}$ laser light for $4 \mathrm{~h}$ (average power of $4 \mathrm{~mW}$ ) and, except for very limited bleaching, we did not observe any changes in absorption, excitation, or emission spectra. However, when the sample was illuminated with $270 \mathrm{~nm}$ laser light using similar excitation power, a decrease in absorbance of the $488 \mathrm{~nm}$ absorption peak of about $17 \%$ accompanied by a blue shift of $2 \mathrm{~nm}$ as well as the appearance of two new absorption bands in the red region of the spectrum (peaks at 563 and $622 \mathrm{~nm}$ ) could be observed. Excitation in these new bands did not give rise to any fluorescence. When the sample was kept in the dark at $4{ }^{\circ} \mathrm{C}$ for $24 \mathrm{~h}$, the absorption spectrum recovered partially and the two bands from the red region of the absorption spectrum disappeared.

Fluorescence Decays and Time-Resolved Emission Spectra (TRES) Excited at $400 \mathrm{~nm}$. The global analysis results of the fluorescence intensity decays excited at $400 \mathrm{~nm}$ in the maximum of the protonated absorption band and detected from 450 to 570 
TABLE 1: Results of the Global Analysis of the Fluorescence Decays Excited at Different Wavelengths

\begin{tabular}{|c|c|c|c|c|c|c|c|c|}
\hline $\begin{array}{c}\text { excitation } \\
(\mathrm{nm})\end{array}$ & $\begin{array}{c}\text { emission } \\
(\mathrm{nm})\end{array}$ & $a_{1}^{\text {rel }}$ & $\begin{array}{c}\tau_{1} \\
\text { (ns) }\end{array}$ & $a_{2}^{\text {rel }}$ & $\begin{array}{c}\tau_{2} \\
(\mathrm{~ns})\end{array}$ & $a_{3}^{\text {rel }}$ & $\begin{array}{c}\tau_{3} \\
(\mathrm{~ns})\end{array}$ & $\chi^{2}$ \\
\hline 400 & $\begin{array}{l}450 \\
480 \\
510 \\
540 \\
570\end{array}$ & $\begin{array}{l}0.731 \\
0.244 \\
0 \\
0 \\
0\end{array}$ & 0.25 & $\begin{array}{l}0.257 \\
0.419 \\
0.498 \\
0.477 \\
0.482\end{array}$ & 2.7 & $\begin{array}{l}0.012 \\
0.337 \\
0.502 \\
0.523 \\
0.518\end{array}$ & 3.4 & 1.09 \\
\hline 420 & $\begin{array}{l}470 \\
475 \\
480 \\
490 \\
500 \\
510 \\
520 \\
530 \\
540 \\
550\end{array}$ & $\begin{array}{l}0.230 \\
0.121 \\
0.062 \\
0.012 \\
0 \\
0 \\
0 \\
0 \\
0 \\
0\end{array}$ & 0.25 & $\begin{array}{l}0.742 \\
0.695 \\
0.650 \\
0.661 \\
0.687 \\
0.717 \\
0.702 \\
0.700 \\
0.696 \\
0.705\end{array}$ & 2.7 & $\begin{array}{l}0.032 \\
0.192 \\
0.296 \\
0.329 \\
0.308 \\
0.279 \\
0.295 \\
0.298 \\
0.301 \\
0.293\end{array}$ & 3.4 & 1.12 \\
\hline 440 & $\begin{array}{l}470 \\
480 \\
490 \\
500 \\
510 \\
520 \\
530 \\
540\end{array}$ & $\begin{array}{l}0.030 \\
0.020 \\
0.015 \\
0.006 \\
0 \\
0 \\
0 \\
0\end{array}$ & 0.25 & $\begin{array}{l}0.71 \\
0.71 \\
0.75 \\
0.80 \\
0.83 \\
0.81 \\
0.81 \\
0.82\end{array}$ & 2.7 & $\begin{array}{l}0.26 \\
0.27 \\
0.23 \\
0.19 \\
0.17 \\
0.19 \\
0.19 \\
0.18\end{array}$ & 3.4 & 1.09 \\
\hline 460 & $\begin{array}{l}490 \\
500 \\
510 \\
520 \\
530 \\
540 \\
550 \\
560 \\
570\end{array}$ & & - & $\begin{array}{l}0.83 \\
0.85 \\
0.89 \\
0.90 \\
0.89 \\
0.90 \\
0.92 \\
0.90 \\
0.89\end{array}$ & 2.7 & $\begin{array}{l}0.170 \\
0.15 \\
0.11 \\
0.10 \\
0.11 \\
0.10 \\
0.08 \\
0.10 \\
0.11\end{array}$ & 3.4 & 1.21 \\
\hline 488 & $\begin{array}{l}500 \\
510 \\
520 \\
530 \\
540 \\
550 \\
560 \\
570\end{array}$ & & - & $\begin{array}{l}0.887 \\
0.819 \\
0.813 \\
0.835 \\
0.845 \\
0.845 \\
0.833 \\
0.834\end{array}$ & 2.7 & $\begin{array}{l}0.113 \\
0.181 \\
0.187 \\
0.165 \\
0.155 \\
0.155 \\
0.167 \\
0.166\end{array}$ & 3.4 & 1.12 \\
\hline 543 & 560 & & & & & 1 & 3.3 & 1.1 \\
\hline
\end{tabular}

$\mathrm{nm}$ with a time increment of $4.88 \mathrm{ps} / \mathrm{ch}$ are summarized in Figure 2. The recorded decays could be fitted using a three exponential model $\left(\chi^{2}=1.09\right)$ with decay times of $0.25,2.7$, and 3.4 ns (Figure 2 and Table 1). The subnanosecond component was detected in the spectral region where the protonated form emits and only with positive contribution to the fluorescence decays. Its amplitude displays the largest value at $450 \mathrm{~nm}$ (about $75 \%$ of the total fluorescence) and decreases rapidly when the detection wavelength increases, disappearing after $500 \mathrm{~nm}$. The same value of $0.25 \mathrm{~ns}$ for this short decay time component was recovered from the global analysis of the fluorescence decays excited at $400 \mathrm{~nm}$ and detected at $450 \mathrm{~nm}$ in different time windows, i.e., using a time increment of 4.88 and $1.49 \mathrm{ps} / \mathrm{ch}$. A corresponding rise time could not be found in the red part of emission spectrum, even by increasing the time resolution up to $1.49 \mathrm{ps} / \mathrm{ch}$. The long $3.4 \mathrm{~ns}$ component, almost absent in the beginning at $450 \mathrm{~nm}$, increases in amplitude until $500 \mathrm{~nm}$ and then maintains a constant level of about 50\% in the red part of the emission spectrum. It is interesting to note the inverse relation, with a crossing over at $480 \mathrm{~nm}$, between the relative amplitudes of the 0.25 and 3.4 ns components (see Figure 2a). The $2.7 \mathrm{~ns}$ component has an almost constant

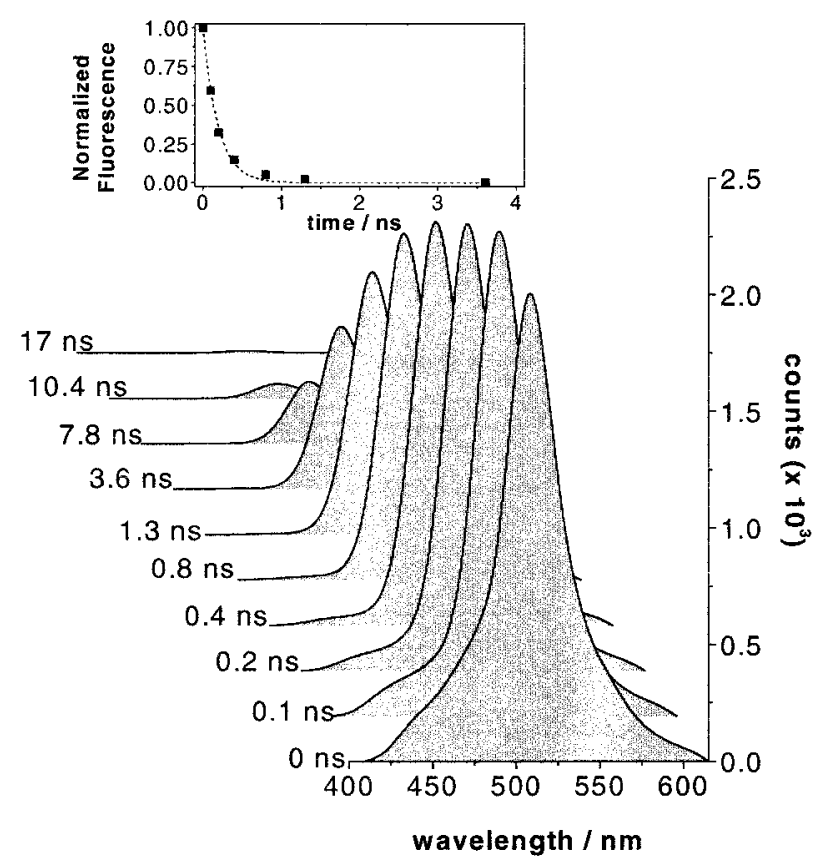

Figure 3. Time-resolved emission spectra of EGFP excited at 400 $\mathrm{nm}$. The inset presents the time evolution of the normalized intensity of the $450 \mathrm{~nm}$ band (full square). An exponential fit of the data (dashed line in inset) results in a time constant of $0.17 \mathrm{~ns}$. The $450 \mathrm{~nm}$ band is the result of the decomposition of the TRES using Gaussian functions.

contribution over the investigated emission range. A deuterated EGFP sample excited at $400 \mathrm{~nm}$ and analyzed at $450 \mathrm{~nm}$ did not show any change in the value of the subnanosecond decay time (data not shown).

The TRESs are displayed in Figure 3. Following the time evolution of the emission spectrum, we can clearly see the disappearance of the $450 \mathrm{~nm}$ band within one nanosecond, whereas the $509 \mathrm{~nm}$ band remains present over the entire time window of the experiment. Decomposition of the detected TRES with the same Gaussian functions used for the stationary emission spectrum upon excitation at $400 \mathrm{~nm}$ allowed us to extract the contribution of the $450 \mathrm{~nm}$ fluorescence band over the investigated time window. A single-exponential fit of the time evolution of the normalized intensity of this band yielded a time constant of about $0.17 \mathrm{~ns}$ (inset in Figure 3). In the limits of the experimental errors, this time constant can be regarded as the $0.25 \mathrm{~ns}$ component detected by global analysis of the fluorescence decays.

Fluorescence Decays Excited at $488 \mathrm{~nm}$. In comparison with the above-described time-resolved experiment, the fluorescence decays recorded upon excitation at $488 \mathrm{~nm}$ and detected from 500 to $570 \mathrm{~nm}$ with a $4.88 \mathrm{ps}$ time increment could be globally analyzed using a two exponential model function $\left(\chi^{2}=1.12\right)$ yielding the same two long decay times of 2.7 and $3.4 \mathrm{~ns}$ detected also by exciting at $400 \mathrm{~nm}$. The results of the analysis are displayed in Figure 4 and Table 1. In the limits of the experimental errors, both relative amplitudes exhibit a constant contribution over the investigated emission range, with an almost $90 \%$ contribution to the total fluorescence for the $2.7 \mathrm{~ns}$ component, which might be a possible indication for two distinct and noninteracting emissive species absorbing at this wavelength.

Fluorescence Decays Excited at 420, 440, and $460 \mathrm{~nm}$. Similar results as those for the $400 \mathrm{~nm}$ time-resolved experiment were obtained from the global analysis of the fluorescence decays excited at $420 \mathrm{~nm}$ and detected from 470 to $550 \mathrm{~nm}$ (see Table 1). A three exponential model function, having the 

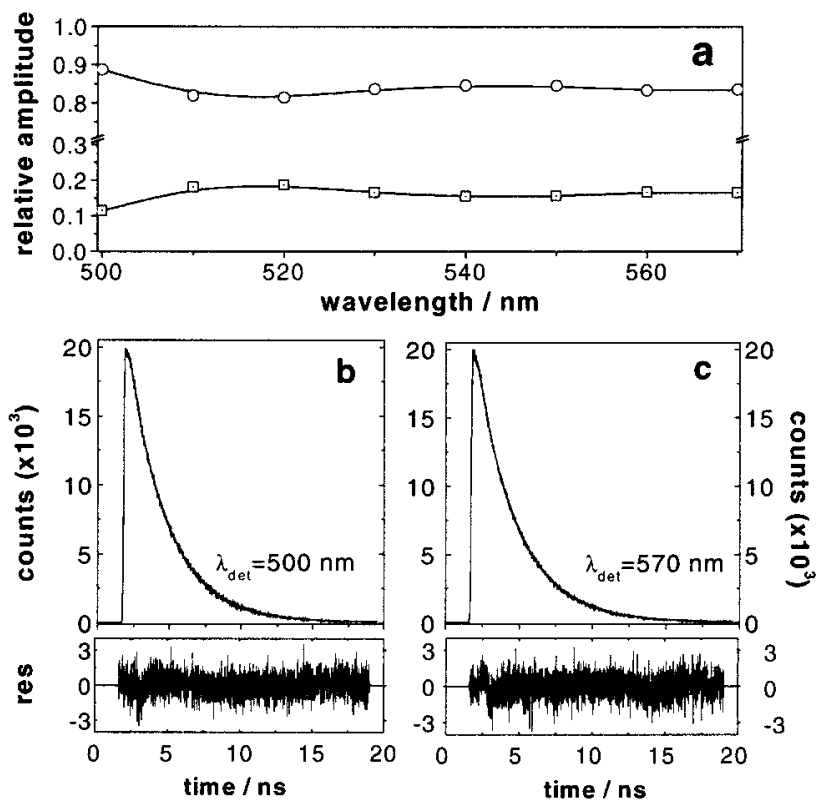

Figure 4. Global analysis results of the fluorescence decays of EGFP excited at $488 \mathrm{~nm}$ and detected from 500 to $570 \mathrm{~nm}$. (a) The relative amplitudes of the 2.7 (line + open circle) and 3.4 ns (line + open square) decay times as a function of emission wavelength. (b) Fit (solid line) and residuals (lower panel) of the fluorescence decay detected at $500 \mathrm{~nm}$. (c) Fit (solid line) and residuals (lower panel) of the fluorescence decay detected at $570 \mathrm{~nm}$.

same decay time constants as those recovered at $400 \mathrm{~nm}$ excitation, was sufficient but necessary to fit the recorded decays. In this case, the amplitude of the $0.25 \mathrm{~ns}$ component has a smaller contribution to the total fluorescence as compared with that of the $400 \mathrm{~nm}$ excitation, disappearing also above 500 $\mathrm{nm}$. The same inverse relation between the relative amplitudes of the 0.25 and $3.4 \mathrm{~ns}$ components was observed for this excitation wavelength. Both 2.7 and 3.4 ns components display a constant contribution for the relative amplitudes above 480 $\mathrm{nm}$. Nevertheless, the relative amplitude of the $2.7 \mathrm{~ns}$ component increased substantially, to about $75 \%$, as compared with $45 \%$ for $400 \mathrm{~nm}$ excitation, whereas the $3.4 \mathrm{~ns}$ component decreases to about $30 \%$ of the total fluorescence. The fluorescence decays excited at $440 \mathrm{~nm}$ and detected from 470 to $540 \mathrm{~nm}$ were globally analyzed also using a three exponential model function having the same decay time components recovered as for 400 $\mathrm{nm}$ excitation (see Table 1). In reference to the relative amplitudes of the two long decay times, the 3.4 ns component contributes at this excitation wavelength about $20 \%$ of the total fluorescence, whereas the $2.7 \mathrm{~ns}$ component contributes about $80 \%$, both with a constant contribution over the detected spectral range. By increasing the excitation wavelength to $460 \mathrm{~nm}$, which is outside the absorption band of the protonated form, the recorded fluorescence decays exhibit only the two long decay time components of 2.7 and 3.4 ns; a two exponential model function being sufficient to fit globally the decays detected from 490 to $570 \mathrm{~nm}$ (Table 1). A decrease in contribution to $15 \%$ of the relative amplitude of the $3.4 \mathrm{~ns}$ component upon excitation at $460 \mathrm{~nm}$ was observed. Also, in this case, both components exhibit a constant contribution over the investigated emission range.

Red Edge Excitation Time-Resolved Experiments. With the excitation of EGFP in the red edge of the absorption spectrum with $543 \mathrm{~nm}$ laser light, the detected fluorescence decay at $560 \mathrm{~nm}$ could be analyzed using a monoexponential model function described by a 3.3 ns time constant (see Table
1), indicating the presence in this spectral region of a single emitting species.

\section{Discussion}

Steady-State Spectroscopy and Photoconversion. Several experimental facts observed from the stationary spectroscopic experiments reported here as well as in previous published papers ${ }^{19,23}$ clearly indicate the presence of the A, B, and I forms in the case of the EGFP mutant. The absorption spectrum of EGFP, sensitive to $\mathrm{pH}$ and exhibiting an isosbestic point at 425 $\mathrm{nm},{ }^{23}$ indicates the presence of two populations in the ground state, with the equilibrium between them being modified by changing the bulk proton concentration. Depending on the excitation wavelength, EGFP exhibits dual emission (see Figure 1) with the minor $450 \mathrm{~nm}$ peak appearing only upon excitation in the $400 \mathrm{~nm}$ absorption band. The major peak from the excitation spectrum exhibits a red shift by increasing the detection wavelength (see Figure 1 lower panel), indicating the presence of two absorbing species above $400 \mathrm{~nm}$. Under these conditions, the protonated A form is responsible for the absorption at $400 \mathrm{~nm}$ and emission at $450 \mathrm{~nm}$, whereas the two deprotonated forms I and B are responsible for the absorption around $488 \mathrm{~nm}$ and the green emission $(509 \mathrm{~nm})$.

The reversible photoconversion taking place in the wild-type GFP between the A, I, and B forms observed by irradiation with $400 \mathrm{~nm}$ light is well-known. ${ }^{8,14}$ The absence of this phenomenon in EGFP could be due to a larger barrier in the ground state between the protonated A and deprotonated B and I forms compared to that of the natural protein. Alternatively, a fast thermal equilibration of the ground states of the different forms of the chromophore at room temperature might be considered. On the other hand, the $270 \mathrm{~nm}$ experiment in which the decrease in absorbance of the major peak at $488 \mathrm{~nm}$ is accompanied by the appearance of two new bands in the red spectral range reveals the existence of a reversible photoconversion process because, after keeping the sample in the dark, the absorption spectrum of the protein recovers partially. This reversible photomodification may be traced back to a change in the structure of the chromophore, e.g., a possible cis-trans photo isomerization that leads to a nonfluorescent species having a red-shifted absorption spectrum. A similar but irreversible photoconversion was reported by Lossau et al. ${ }^{15}$ for wild-type GFP when illuminated with $400 \mathrm{~nm}$ laser light.

Relying on the stationary data reported here as well as on the work of Völker and co-workers, ${ }^{19}$ who identified the three forms A, I, and B together with their $0-0$ transitions in holeburning experiments at a cryogenic temperature for the closely related S65T mutant $(429,478$, and $495 \mathrm{~nm}$ for the A, B, and I forms, respectively), the absorption spectrum of EGFP can be decomposed, using Gaussian functions, into three absorption bands corresponding to the A, B, and I forms, respectively, as depicted in Figure 1.

Time-Resolved Fluorescence Spectroscopy. As mentioned in the Introduction, previous time-resolved fluorescence studies explained the excited-state dynamics of EGFP using a similar decay scheme with three levels and implying ESPT as for the wild-type GFP. As a consequence of this model, the two long decay times detected in our experiments would reflect the depopulation of the $\mathrm{B}^{*}$ and $\mathrm{I}^{*}$ states, similar to the report of Stryker et al. ${ }^{16}$ for the wild-type GFP (2.8 and $3.3 \mathrm{~ns}$ fluorescence decay times for the $\mathrm{B}^{*}$ and $\mathrm{I}^{*}$ states). With a reliance on the decomposition of the absorption spectrum, the detection of a single component of $\sim 3.3 \mathrm{~ns}$ at $560 \mathrm{~nm}$ by exciting in the red edge of the absorption spectrum at $543 \mathrm{~nm}$ 


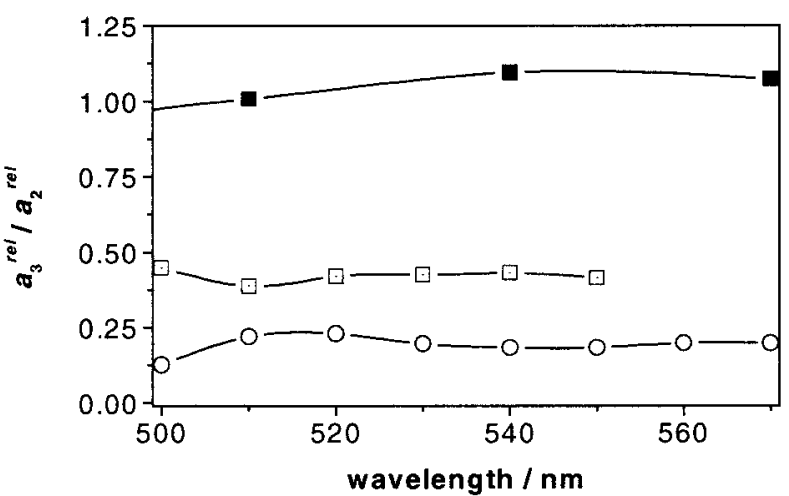

Figure 5. Ratio of the relative amplitudes $a_{3}^{\text {rel }}$ and $a_{2}^{\text {rel }}$ of the 3.4 and $2.7 \mathrm{~ns}$ components as a function of emission wavelengths for timeresolved experiments performed at 400 (line + full square), 420 (line + open square), and $488 \mathrm{~nm}$ (line + open circle) excitation.

mainly reflects the fluorescence decay time of the I* state, whereas the $2.7 \mathrm{~ns}$ component mainly reflects the depopulation of the $\mathrm{B}^{*}$ state. Consequently, in both absorption and emission spectra, the I form is red shifted as compared with the B one, similar to that as reported by Völker and co-workers ${ }^{19}$ from hole burning experiments. Because of the absence of the 0.25 ns component and the constant contribution of the 2.7 and 3.4 ns components over the whole emission range upon excitation at 460 and $488 \mathrm{~nm}$, the $\mathrm{I}^{*}$ and $\mathrm{B}^{*}$ forms can be regarded as independently emissive species with overlapping spectra but different fluorescence lifetimes of 3.4 and $2.7 \mathrm{~ns}$, respectively. The 0.25 ns component, present in our experiments only as a positive contribution in the fluorescence decays, was detected only below $500 \mathrm{~nm}$ emission wavelength and by exciting into the absorption band of the A form. A similar subnanosecond component was also detected by Volkmer et al. ${ }^{17}$ and interpreted as an average value of multiple decay time components. Noticing the disappearance within $1 \mathrm{~ns}$ of the $450 \mathrm{~nm}$ band in the TRES (see Figure 3) as well as the absence of a deuteration effect on the subnanosecond component as well as the absence of a corresponding rise time in the red part of the emission spectrum, we can associate this decay time with the deactivation of the A* state. Moreover, ESPT taking place in $0.25 \mathrm{~ns}$ would be very improbable because EGFPs chromophore has a similar structure and environment as that in the wild-type GFP where the same process takes place in 4 ps. Furthermore, a 10 ps decay time which slows down by deuteration was already reported for EGFP and was associated with ESPT. ${ }^{15}$ Because both I* and $\mathrm{B} *$ states of the chromophore exhibit fluorescence decay times of about $3 \mathrm{~ns}$, the $0.25 \mathrm{~ns}$ component corresponding to the deactivation of the $\mathrm{A}^{*}$ state has to be regarded as fluorescence quenched by a nonradiative process, other than ESPT.

However, the time-resolved data presented in this contribution proves that the $I^{*}$ state is at least partially populated via an excited-state reaction when exciting EGFP with $400 \mathrm{~nm}$ light. Indeed, at $400 \mathrm{~nm}$, a wavelength very improbable for the direct excitation of the I form (see Figure 1), the contribution of the $I^{*}$ state to the total fluorescence detected above $500 \mathrm{~nm}$ is larger (about 50\%) compared to the excitation at $488 \mathrm{~nm}$ (about 15\%). Moreover, as one can see from Figure 5 , the ratio $a_{3}^{\text {rel }} / a_{2}^{\text {rel }}$ of the relative amplitudes of the 3.4 and 2.7 ns decay times decreases in value by increasing the excitation wavelength, different that one expects from the case when both $\mathrm{I}^{*}$ and $\mathrm{B}^{*}$ species would emit independently, with the I form being spectrally red shifted. Finally, the mirror symmetry observed between the relative amplitudes of the 0.25 and 3.4 ns components (see Figure 2)

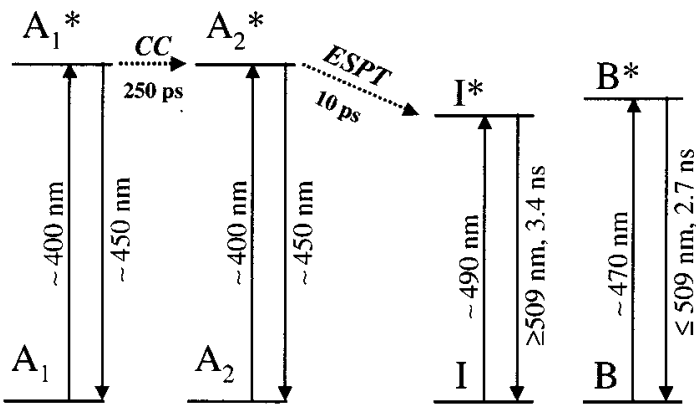

Figure 6. Model proposed for EGFP's excited-state dynamics on the basis of this work and previous published data: ${ }^{15,19,23} \mathrm{~A}_{1}$ and $\mathrm{A}_{2}$, protonated forms of the chromophore; I and $\mathrm{B}$, deprotonated forms of the chromophore; CC, conformational change; ESPT, excited-state proton transfer. The model does not take into account eventual processes between the ground state forms of the chromophore.

can be explained by an excited-state reaction involving both a precursor state absorbing at $400 \mathrm{~nm}$ and the I* state. Alternatively, one can consider the presence of two independent emissive species with sufficiently separated spectra, one responsible for the fluorescence decaying with $0.25 \mathrm{~ns}$ and the other one the I* species. However, upon excitation at $400 \mathrm{~nm}$, the green fluorescence of EGFP is the result of the emission from both $\mathrm{I}^{*}$ and $\mathrm{B} *$ states with similar contributions to the total detected fluorescence, different than for the wild-type GFP, where the $I^{*}$ state mainly contributes to the green fluorescence. ${ }^{16}$

Photophysical Scheme for EGFP. The data reported here together with that of previously published experiments cannot be fully explained taking into account a decay scheme with only three levels, similar to that proposed for the wild-type GFP and adapted to EGFP. ${ }^{15}$ At least one additional protonated state has to be considered.

We suggest a modified photophysical scheme ${ }^{36}$ containing two protonated forms $\mathrm{A}_{1}$ and $\mathrm{A}_{2}$, both absorbing at around 400 $\mathrm{nm}$ and emitting at around $450 \mathrm{~nm}$, as well as the two deprotonated B and I forms (Figure 6).

In this model, direct excitation at $400 \mathrm{~nm}$ leads to a conformationally unrelaxed protonated state $\mathrm{A}_{1} *$ (although direct excitation to $\mathrm{A}_{2} *$ cannot be excluded), which can decay radiatively to the corresponding ground-state $\mathrm{A}_{1}$ or can relax nonradiatively to the $\mathrm{A}_{2}$ * state in subnanosecond time, undergoing a conformational change of the chromophore. The $\mathrm{A}_{2} *$ state will be depopulated by radiative deactivation to the corresponding ground-state $\mathrm{A}_{2}$ or by ESPT to the I* state, with the last process taking place in about $10 \mathrm{ps}$ and being the dominant relaxation channel. ${ }^{15}$ From this point, processes similar to those of wild-type GFP take place: I* will be deactivated mainly by direct relaxation to the ground state I and with low probability to the relaxed $\mathrm{B}^{*}$ state. In principle, for such a model, upon excitation at $400 \mathrm{~nm}$, the fluorescence decay detected above $500 \mathrm{~nm}$ should contain the $0.25 \mathrm{~ns}$ component with a negative contribution and the $10 \mathrm{ps}$ component with a positive contribution. This is valid only when spectral overlap between the different emissive forms is excluded. When the species that contribute to the detected fluorescence overlap in emission, like in the case of the $\mathrm{I}^{*}$ and $\mathrm{B}^{*}$ states, the negative contribution of the subnanosecond component can decrease to a value that is not detectable even performing experiments such as those presented in this contribution. In addition, the small population of the protonated species that are excited in EGFP at $400 \mathrm{~nm}$ contributes to the absence of a corresponding subnanosecond rise time. Furthermore, the time resolution of our setup together with the previous mentioned facts will also account for the absence of a 10 ps component. The existence of this $10 \mathrm{ps}$ 


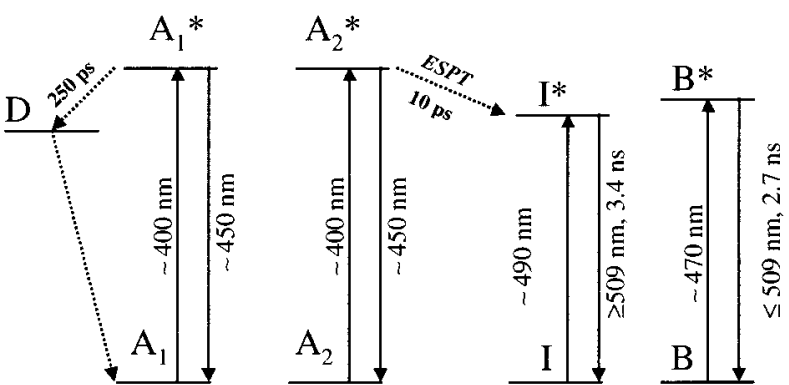

Figure 7. Alternative model to the one proposed in Figure 6 for EGFPs excited-state dynamics on the basis of this work and previous published data: ${ }^{15,19,23} \mathrm{~A}_{1}$ and $\mathrm{A}_{2}$, protonated forms of the chromophore; I and $\mathrm{B}$, deprotonated forms of the chromophore; D, dark state; ESPT, excitedstate proton transfer. The model does not take into account eventual processes between the ground state forms of the chromophore.

component, influenced by deuteration, was demonstrated for EGFP by Lossau et al. ${ }^{15}$ with fluorescence upconversion experiments. The $A_{1} *-A_{2} *$ relaxation process, regarded here as a conformational change of the chromophore, probably can be induced by a change in the surrounding hydrogen bond network. It has been shown that, upon excitation, such a conformational relaxation process can take place in proteins in the subnanosecond time scale. ${ }^{37,38}$ Recently, also theoretical calculations predicted the existence of at least two protonated forms of the chromophore in GFP proteins responsible for absorption at $400 \mathrm{~nm} .{ }^{39}$

Alternatively, a model describing the photophysical processes in EGFP as that depicted in Figure 7 can be considered. ${ }^{36}$ In this figure, the $A_{1}$ and $A_{2}$ forms can be regarded as two subpopulations of the protein with two different conformations of the protonated chromophore. In this model, upon excitation at $400 \mathrm{~nm}$, two corresponding excited-state protonated forms $\mathrm{A}_{1} *$ and $\mathrm{A}_{2} *$ will be created. The $\mathrm{A}_{2} *$ form, having a conformation that allows immediately ESPT, will relax to the $I^{*}$ state within $10 \mathrm{ps}$. The $\mathrm{A}_{1} *$ form will decay radiatively to the corresponding ground-state $\mathrm{A}_{1}$ within $0.25 \mathrm{~ns}$, with the fluorescence being quenched by a competitive radiationless deactivation process. Eventually, this radiationless deactivation process is a cis-trans isomerization of the chromophore as predicted by Weber et al. ${ }^{22}$ from quantum chemical calculations.

The results presented in this contribution do not give a direct spectroscopic proof of the existence of the second protonated form of the chromophore, but in combination with previous published experiments, the introduction of this additional protonated form may explain the complicated photophysics of EGFP.

\section{Conclusions}

We have systematically investigated, using stationary and picosecond fluorescence time-resolved experiments, the photophysical properties of the EGFP variant carrying Ser65-Thr and Phe64-Leu mutations. The global analysis of the fluorescence decays excited at different wavelengths between 400 and $543 \mathrm{~nm}$ and detected over the emission range of the protein allowed us to determine the fluorescence decay times of the $I^{*}$ (3.4 ns) and $\mathrm{B}^{*}(2.7 \mathrm{~ns})$ deprotonated states of the chromophore. The subnanosecond component detected upon excitation at 400 $\mathrm{nm}$ was attributed to quenched fluorescence emitted from the protonated excited state. To explain the presence of this subnanosecond component and of the previously reported 10 ps component, an additional protonated form of the chromophore has to be taken into account. Two alternative models were proposed to explain the excited-state dynamics taking place in EGFP. Both models can also be applied to the wild-type GFP. Indeed, a subnanosecond decay time of 0.12 ns was reported by Boxer et al. ${ }^{14}$ for wild-type GFP, being present only when exciting at $400 \mathrm{~nm}$ and detecting at $450 \mathrm{~nm}$, without a corresponding rise time at $508 \mathrm{~nm}$ and, like in the case of EGFP, not affected by deuteration of the protein.

Acknowledgment. The authors gratefully acknowledge the F.W.O, the Flemish Ministry of Education for the support through GOA/1/96, the support of DWTC (Belgium) through IUAP-IV-11, and the support of the K. U. Leuven through an IDO project. J.H. thanks the F.W.O for a postdoctoral fellowship. M.C. thanks K. U. Leuven for a doctoral fellowship.

\section{References and Notes}

(1) Chalfie, M.; Euskirchen, Tu. G.; Ward, W. W.; Prasher, D. C. Science 1994, 263, 802 .

(2) Tsien, R. Y. Апnu. Rev. Biochem. 1998, 67, 509

(3) Chalfie, M. Green fluorescent protein, properties, applications and protocols; Wiley-Liss, Inc: New York, 1998.

(4) Presley, J. F.; Cole, N. B.; Schroer, T. A.; Hirschberg, K.; Zaal, K. J.; Lippincott-Schwartz, J. Nature 1997, 389, 81.

(5) Lee, S.; Neumann, M.; Stearman, R.; Stauber, R.; Pause, A.; Pavlakis, G. N.; Klausner, R. D. Mol. Cell. Biol. 1999, 19, 1486.

(6) Stauber, R. H.; Afonina, E.; Gulnik, S.; Erikson, J.; Pavlakis, G. N. Virology 1998, 251, 38.

(7) Cody, C. W.; Prasher, D. C.; Westler, W. M.; Prendergast, F. G.; Ward, W. W. Biochemistry 1993, 32, 1212.

(8) Cubbit, A. B.; Heim, R.; Adams, S. R.; Boyd, A. E.; Gross, L. A.; Tsien, R. Y. Trends Biochem. Sci. 1995, 20, 448.

(9) Ormö, M.; Cubitt, A. B; Kallio, K.; Gross, L. A.; Tsien, R. Y.; Remington, S. J. Science 1996, 273, 1392.

(10) Yang, F.; Moss, L. G.; Philips, G. N. Nat. Biotechnol. 1996, 14, 1246

(11) Brejc, K.; Sixma, T. K.; Kitts, P. A.; Kain, S. R.; Tsien, R. Y. Proc. Natl. Acad. Sci. U.S.A. 1997, 94, 2306.

(12) Heim, R.; Prasher, D. C.; Tsien, R. Y. Proc. Natl. Acad. Sci. U.S.A 1994, 91, 12501 .

(13) Heim, R.; Cubitt, A. B.; Tsien, R. Y. Nature 1995, 373, 663.

(14) Chattoraj, M.; King, B. A.; Bublitz, G. U.; Boxer, S. G. Proc. Natl. Acad. Sci. U.S.A. 1996, 93, 8362.

(15) Lossau, H.; Kummer, R. A.; Heinecke, F.; Pöllinger-Dammer, C.; Kompa, G.; Bieser, J. T.; Silva, C. M.; Yang, M. M.; Youvan, D. C.; MichelBeyerle, M. E. Chem. Phys. 1996, 213, 1.

(16) Stryker, G.; Subramanian, V.; Seidel, C. A. M.; Volkmer, A. J. Phys. Chem. B 1999, 103, 8612.

(17) Volkmer, A.; Subramanian, V.; Birch, D. J. S.; Jovin, T. M. Biophys. J. 2000, 78, 1589 .

(18) Creemers, T. M. H.; Lock, A. J.; Subramanian, V.; Jovin, T. M.; Völker, S. Nat. Struct. Biol. 1999, 6, 557.

(19) Creemers, T. M. H.; Lock, A. J.; Subramanian, V.; Jovin, T. M.; Völker, S. Proc. Natl. Acad. Sci. U.S.A. 2000, 97, 2974

(20) Voityuk, A. A.; Michel-Beyerle, M. A.; Rosch, N. Chem. Phys Lett. 1998, 231, 13.

(21) Voityuk, A. A.; Michel-Beyerle, M. A.; Rosch, N. Chem. Phys. Lett. 1998, 296, 269.

(22) Weber, W.; Helms, V.; McCammon, J. A.; Langhoff, P. W. Proc. Natl. Acad. Sci. U.S.A. 1999, 96, 6177

(23) Haupts, U.; Maiti, S.; Schwille, P.; Webb, W. W. Proc. Natl. Acad. Sci. U.S.A. 1998, 95, 13573.

(24) Ward, W. W.; Prentice, H. J.; Roth, A. F.; Cody, C. W.; Reeves, S. C. Photochem. Photobiol. 1982, 35, 803.

(25) Ward, W. W.; Bokman, S. H. Biochemistry 1982, 21, 4535.

(26) Van Thor, J. J.; Pierik, A. J.; Nugteren-Roodzant, I.; Xie, A.; Hellingwerf, K. J. Biochemistry 1998, 37, 16915.

(27) Widengren, J.; Terry, B.; Rigler, R. Chem. Phys. 1999, 249, 259

(28) Widengren, J.; Mets, U.; Rigler, R. Chem. Phys. 1999, 250, 171.

(29) Sambrook, J.; Fritsch, E. F.; Maniatis, T. Molecular cloning: A Laboratory Manual, 2nd ed; Cold Spring Harbor Laboratory Press: Spring Harbor, NY, 1989.

(30) Lakowicz, J. R. Principles of Fluorescence Spectroscopy; Plenum Press: New York, 1986.

(31) Maus, M.; Rousseau, E.; Cotlet, M.; Hofkens, J.; Schweitzer, G.; Van der Auweraer, M.; De Schryver, F. C.; Kruegen, A. Rev. Sci. Instrum 2001, 72, 1, 36 . 
(32) Zuker, M.; Szabo, A. G.; Bramall, L.; Krajcarski, D. T.; Selinger,

B. Rev. Sci. Instrum. 1985, 56, 14.

(33) Janssens, L. D.; Boens, N.; Ameloot, M.; De Schryver, F. C. J. Phys. Chem. 1990, 94, 3564.

(34) Van den Zegel, M.; Boens, N.; Daems, D.; De Schryver, F. C. Chem. Phys. 1986, 101, 311.

(35) Hofkens, J. Ph.D. Thesis, Katholieke Universiteit Leuven, 1993.

(36) In both models proposed in Figures 6 and 7, the eventual interconversions between the deprotonated forms of the chromophore as well as the displacement of different ground states are not discussed because no data are available in the literature for the EGFP mutant.

(37) Bastiaens, P. I. H.; Van Hoek, A.; Van Berkel, W. J. H.; De Kok, A.; Visser, A. J. G. W. Biochemistry 1992, 31, 7061.

(38) Demchenko, A. P. Eur. Biophys. J. 1988, 16, 121.

(39) Yazal, J. E.; Prendergast, F. G.; Shaw, D. E.; Pang, Y. P. J. Am. Chem. Soc. 2000, 122, 11411. 